\title{
Meal and snack frequency in relation to diet quality in US children and adolescents: the National Health and Nutrition Examination Survey 2003-2012
}

\author{
Kentaro Murakami ${ }^{1, *}$ and M Barbara E Livingstone ${ }^{2}$ \\ 'Department of Nutrition, School of Human Cultures, University of Shiga Prefecture, Hikone, Shiga 522 8533, \\ Japan: ${ }^{2}$ Northern Ireland Centre for Food and Health, Ulster University, Coleraine, UK
}

Submitted 1 October 2015: Final revision received 4 December 2015: Accepted 6 January 2016: First published online 26 February 2016

\begin{abstract}
Objective: To examine the associations of meal frequency (MF) and snack frequency (SF) with diet quality.

Design: Dietary intake was assessed using two $24 \mathrm{~h}$ dietary recalls. All eating occasions providing $\geq 210 \mathrm{~kJ}$ of energy were divided into meals or snacks on the basis of contribution to energy intake $(\geq 15 \%$ or $<15 \%)$, self-report and time (06.00-09.00, 12.00-14.00 and 17.00-20.00 hours, or others). Diet quality was assessed using the Healthy Eating Index (HEI)-2010.

Setting: Nationally representative sample of the US population.

Subjects: Children aged $6-11$ years $(n$ 4269) and adolescents aged $12-19$ years ( $n$ 6193) in the National Health and Nutrition Examination Survey 2003-2012.

Results: Irrespective of the definition of meals, higher MF was associated with higher HEI-2010 in both children and adolescents. One additional meal per day increased HEI-2010 by $1.45-3.59$ points (all $P<0.005$ ). Conversely, the associations for SF were inconsistent. While SF based on energy contribution was positively associated with HEI-2010 in both children and adolescents $(0.70(P=0.001)$ and $1.00(P<0.0001)$ point increase by one additional snack, respectively), there were no associations for SF based on self-report or time. In analyses in which only plausible energy reporters (3425 children and 3753 adolescents) were included, similar results were obtained.

Conclusions: In a representative sample of US children and adolescents, MF was associated with better diet quality, while the associations for SF varied depending on the definition of snacks. The findings highlight the importance of applying different definitions of meals and snacks when assessing the impact of dietary patterns on health.
\end{abstract}

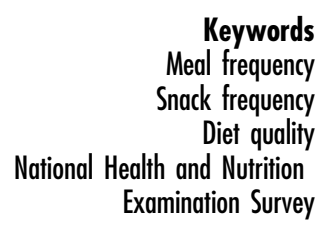

Diet in childhood and adolescence has been suggested to have a potential lifelong effect on the development of many chronic diseases such as obesity ${ }^{(1)}$ and $\mathrm{CVD}^{(2)}$. Additionally, evidence suggests that dietary patterns established during childhood and adolescence persist into adulthood at least to some extent ${ }^{(3,4)}$. Thus, investigation of dietary behaviours that contribute to healthier dietary patterns in children and adolescents is a high public health priority. In this regard, eating frequency (EF), i.e. sum of meal frequency (MF) and snack frequency (SF), has received increased attention because of an increasing trend in EF in representative samples of US children and adolescents between the 1970 s and $2000 s^{(5)}$, in parallel with an increasing prevalence of overweight and obesity $^{(6)}$. Nevertheless, research on the association between EF and dietary intake is extremely limited in young populations ${ }^{(7)}$.

Investigation of EF in relation to dietary intake in adult populations has also produced inconsistent associations ${ }^{(8-14)}$. However, several methodological limitations make it difficult to interpret these studies. First, EF has often been estimated using a series of self-report questions $^{(8-11,15-19)}$, the validity of which has not been examined or reported. Only a limited number of studies have estimated $\mathrm{EF}$ with the use of information on actual dietary behaviours (based on dietary record or $24 \mathrm{~h}$ recall) ${ }^{(20-24)}$. Second, there is no consensus about what constitutes a snack, a meal or an eating occasion. While some studies have relied on respondents' self-identification of meals, snacks or eating occasions ${ }^{(8-11,15-18,25,26)}$, others have tried 
to apply more objective criteria ${ }^{(19-24,27-30)}$. Third, the associations between EF and dietary intake may be confounded by the under-reporting of EF accompanied by the under-reporting of energy intake (EI), particularly by obese or overweight individuals ${ }^{(12,13)}$. Taken together, the discrepant findings are not surprising and merit more robust data analyses than hitherto to resolve this issue.

Furthermore, potentially different effects of MF and SF have not been investigated using different definitions of meals and snacks. An accurate distinction between meals and snacks is important, because they are hypothesized to exert different effects on EI and dietary intake ${ }^{(14)}$. This is also important for the development of science-based recommendations on snack and meal patterns for consumers ${ }^{(31)}$. Moreover, in the absence of a universally accepted definition of meals and snacks, an understanding of the influence of different meal and snack definitions on the associations with diet quality may facilitate the interpretation of the existing literature and help establish consensus on the most appropriate research definition for meals and snacks ${ }^{(14)}$.

The aim of the present cross-sectional study in a representative sample of US children and adolescents based on the data from the National Health and Nutrition Examination Survey (NHANES) was to examine the relationship of MF and SF with diet quality, by focusing on the confounding of EI misreporting and the use of different definitions of meals and snacks.

\section{Methods}

\section{Survey design and analytic sample}

The present cross-sectional analysis was based on public domain data from NHANES, a continuing populationbased survey that uses a complex, stratified multistage probability sample design to create a representative sample of the non-institutionalized civilian US population $^{(32,33)}$. The survey examines about 5000 persons each year and the data are released in two-year cycles. The unweighted response rates for the examined persons aged 6-19 years for NHANES 2003-2004, 2005-2006, 2007-2008, 2009-2010 and 2011-2012 were 84\%, 83\%, $83 \%, 86 \%$ and $77 \%$, respectively ${ }^{(34)}$. The documentation and data for each of these surveys used were downloaded from the NHANES website ${ }^{(35)}$.

The analytic sample was limited to children and adolescents aged 6-19 years with two complete and reliable, self-reported $24 \mathrm{~h}$ dietary recall data determined by the National Center for Health Statistics ( $n$ 11712). After excluding pregnant ( $n$ 96) and lactating ( $n$ 15) respondents as well as those with missing information on the variables of interest ( $n 672$ for family poverty income ratio; $n 446$ for education of household head; $n 153$ for body height or weight; $n 3$ for some EF variables; some respondents had more than one missing value), the final analytic sample included 10462 respondents from NHANES 2003-2012.

\section{Dietary assessment}

All surveys collected dietary information with the use of two $24 \mathrm{~h}$ dietary recalls (one by face-to-face interview and one by telephone $3-10 \mathrm{~d}$ after the first recall). The dietary data were collected with the use of an automated five-step multiple pass approach, namely the US Department of Agriculture's Automated Multiple-Pass Method ${ }^{(35-39)}$. Participants were asked to report the time each food and beverage was eaten and to classify each eating occasion from a predefined list of categories, which were used to define meals and snacks, as described later. Proxies, most commonly a parent, assisted with the dietary interview for children aged 6-11 years; dietary intake was self-reported by adolescents aged 12-19 years. Estimates of intakes of energy and selected nutrients from all reported foods and beverages were calculated by using the US Department of Agriculture's Food and Nutrient Database for Dietary Studies ${ }^{(35)}$. The mean of dietary intake over the $2 \mathrm{~d}$ for each participant was used for the present analysis.

\section{Assessment of diet quality}

As a measure of diet quality, the Healthy Eating Index (HEI)-2010 was applied. Food group and nutrient component standards and the development and evaluation of the HEI-2010 have been described previously ${ }^{(40,41)}$. Briefly, the HEI-2010 is an index that reflects conformance/adherence to the 2010 Dietary Guidelines for Americans $^{(42)}$, including twelve components. Of these, nine components assess adequacy of the diet (total fruit; whole fruit; total vegetables; greens and beans; whole grains; dairy; total protein foods; seafood and plant proteins; fatty acids as the ratio of PUFA and MUFA to SFA), with higher scores reflecting better diet quality. The remaining three components assess moderation of the diet (refined grains; sodium; empty calories, i.e. energy from solid fats, alcohol and added sugars), with higher scores reflecting better diet quality (lower consumption). A density approach is used to set standards (i.e. per $4184 \mathrm{~kJ}$ of energy or as a percentage of energy). The scores of the twelve components are summed to a total score (i.e. HEI-2010), the maximum of which is 100. The SAS code used to calculate HEI-2010 was downloaded from the Center for Nutrition Policy and Promotion's website ${ }^{(43)}$.

\section{Definition of eating frequency, meal frequency and snack frequency}

Data from the two $24 \mathrm{~h}$ dietary recalls were also used to calculate the average number of eating occasions per day, i.e. EF. Eating occasions were defined as any occasion when any food or drink was consumed ${ }^{(24-26,30)}$. In many previous studies, if two eating occasions occurred in $\leq 15 \mathrm{~min}$, both events were counted as a single eating occasion; when $>15$ min separated two eating occasions, these were considered distinct eating occasions $^{(20,21,23,24,30)}$. In the present study, however, all foods 
and beverages reported at one discrete clock time were considered as part of one eating occasion, because almost all eating episodes (>99.5\%) occurred at least $15 \mathrm{~min}$ apart in NHANES ${ }^{(44)}$. EF was calculated based on all eating occasions except for those providing $<210 \mathrm{~kJ}$ of energy. This calculation method has been used in several previous studies $^{(21,24-26,30)}$ and was chosen to avoid giving undue weight to eating occasions that included only water, low-calorie beverages or small quantities of foods.

All eating occasions were divided into either meals or snacks with the use of three different published definitions: on the basis of (i) contribution to total $\mathrm{EI}^{(45)}$; (ii) selfreported name of eating occasion ${ }^{(44)}$; and (iii) clock time $^{(46)}$. For the first definition ${ }^{(45)}$, a meal was defined as any eating episode comprising $\geq 15 \%$ of total EI, regardless of the time of day or composition of foods or beverages consumed. All other eating episodes were classified as a snack. For each participant, MF and SF determined based on percentage contribution to total EI were thus calculated (hereafter referred to as $\mathrm{MF}_{\text {energy } \%}$ and $\mathrm{SF}_{\text {energy\%}}$, respectively). For the second definition ${ }^{(4)}$, any eating occasions with self-reported name of 'breakfast', 'brunch', 'lunch', 'supper' and 'dinner' or their equivalents in Spanish were considered meals. All other self-reported eating events (i.e. 'snack', 'drink' and 'extended consumption' or their equivalents in Spanish, as well as 'other' and 'don't know') were considered snacks. For each participant, MF and SF determined based on self-report were thus calculated (hereafter referred to as $\mathrm{MF}_{\text {self-report }}$ and $\mathrm{SF}_{\text {self-report }}$, respectively). For the third definition $^{(46)}$, meals were defined as eating events reported during selected times of the day, i.e. 06.00-09.00, 12.00-14.00 and 17.00-20.00 hours. All other eating occasions were considered snacks. For each participant, MF and SF determined based on the time consumed were thus calculated (hereafter referred to as $\mathrm{MF}_{\text {time }}$ and $\mathrm{SF}_{\text {time }}$, respectively).

\section{Assessment of non-dietary variables}

Race/ethnicity was categorized as non-Hispanic white, non-Hispanic black, Mexican American, and others. As indicators of socio-economic status, we considered family income as a percentage of the federal poverty threshold and years of education of the household head. The family poverty income ratio was categorized as $<130 \%$, $130-349 \%$ and $\geq 350 \%{ }^{(47)}$. The educational level of the household head was categorized as $<12$ years, 12 years, some college, and college degree or more. Information on household size $(\leq 2,3-4$ or $\geq 5)$ was also collected. The physical activity variable was created as follows. For children aged 6-11 years, the response to the question on the number of times per week that play or exercise was hard enough to induce sweat in the past $7 \mathrm{~d}$ (NHANES 2009-2012) or without a specified period (NHANES 2003-2008): 0-3 times $=$ low; $4-6$ times $=$ moderate; and 7 times $=$ active. For adolescents aged $12-19$ years, the responses to two different questions on any leisure-time moderate or vigorous activities lasting $\geq 10 \mathrm{~min}$ in the past $30 \mathrm{~d}$ (NHANES 2003-2006) or without a specified period (NHANES 2007-2012): no to both two questions = low; yes to one question=moderate; and yes to both two questions $=$ active. The hours of screen time were determined from questions on television/video watching $(\mathrm{h} / \mathrm{d})$ or computer use $(\mathrm{h} / \mathrm{d}$ ) over the past $30 \mathrm{~d}$ (except for adolescents aged 12-19 years in NHANES 2007-2008 and 2009-2010, for whom information on sedentary activity was used), which were categorized as $<2, \geq 2$ to $<4, \geq 4$ to $<6$ and $\geq 6 \mathrm{~h} / \mathrm{d}$. BMI $\left(\mathrm{kg} / \mathrm{m}^{2}\right)$ was calculated as weight (in kilograms) divided by height (in metres) squared. The percentile of BMI-for-age was calculated using the SAS program for growth charts available from the Centers for Disease Control and Prevention ${ }^{(48,49)}$. Weight status was defined based on the percentile of BMI-for-age as follows ${ }^{(50)}$ : underweight $(<5$ th percentile); normal $(\geq 5$ th to $<85$ th percentile); overweight $(\geq 85$ th to $<95$ th percentile); and obese ( $\geq 95$ th percentile).

Misreporting of EI was evaluated based on the ratio of EI to estimated energy requirement (EER). EER was calculated using sex-, age- and weight status-specific equations published from the US Dietary Reference Intakes, based on sex, age, body height and weight, and physical activity ${ }^{(51)}$. Because of a lack of an objective measure of physical activity in the present study, we assumed 'low active' level of physical activity (i.e. physical activity level $\geq 1.4$ to $<1 \cdot 6)^{(51)}$ for all participants during this calculation. The $95 \%$ confidence limits of the expected EI:EER of 1.0 (i.e. 0 on the natural log scale) were calculated, taking into account coefficients of variation in intakes and other components of energy balance (i.e. the within-subject variation in $\mathrm{EI}=23 \%$; the error in the EER equations $=4.8 \%$; the day-to-day variation in total energy expenditure $=8 \cdot 2 \%)^{(52)}$. Consequently, under-, acceptable and over-reporters were defined as having EI:EER $<0.69$, $0.69-1.46$ and $>1.46$, respectively.

\section{Statistical analysis}

Statistical analyses were performed for children aged 6-11 years $(n 4269)$ and adolescents aged $12-19$ years ( $n$ 6193) separately, using the statistical software package SAS version 9.2. All of the analyses used the NHANESprovided sampling weights that were calculated to take into account unequal probabilities of selection resulting from the sample design, non-response and planned oversampling of selected subgroups, so that the results are representative of the US community-dwelling population ${ }^{(33,53)}$. Linear regression analyses were performed to explore the associations of $\mathrm{EF}, \mathrm{MF}$ and $\mathrm{SF}$ (independent variables) with dietary intake variables (dependent variables). EF, MF and SF were analysed continuously after confirming the linearity of relationships using tertile, quartile and quintile categories. With the use of the PROC SURVEYREG procedure, we calculated the adjusted 
Table 1 Basic characteristics of the sample of US children and adolescents: National Health and Nutrition Examination Survey 2003-2012*

\begin{tabular}{|c|c|c|c|c|c|}
\hline & \multicolumn{2}{|c|}{ Children aged $6-11$ years $(n$ 4269) } & \multicolumn{2}{|c|}{ Adolescents aged $12-19$ years ( $n$ 6193) } & \multirow[b]{2}{*}{$P \dagger$} \\
\hline & Mean & SE & Mean & SE & \\
\hline EF (times/d) & $4 \cdot 76$ & 0.03 & $4 \cdot 13$ & 0.03 & $<0.0001$ \\
\hline $\mathrm{MF}_{\text {energy\% }}($ times $/ \mathrm{d}) \ddagger$ & 2.87 & 0.01 & 2.68 & 0.01 & $<0.0001$ \\
\hline $\mathrm{SF}_{\text {energy\% }}($ times $/ \mathrm{d}) \ddagger$ & 1.89 & 0.03 & 1.45 & 0.03 & $<0.0001$ \\
\hline $\mathrm{MF}_{\text {self-report }}($ times/d)§ & 2.88 & 0.01 & 2.55 & 0.01 & $<0.0001$ \\
\hline $\mathrm{SF}_{\text {self-report }}($ times $/ \mathrm{d}) \S$ & 1.89 & 0.03 & 1.58 & 0.03 & $<0.0001$ \\
\hline $\mathrm{MF}_{\text {time }}($ times/d)\| & 2.96 & 0.02 & 2.40 & 0.02 & $<0.0001$ \\
\hline $\mathrm{SF}_{\text {time }}($ times/d)\| & 1.80 & 0.02 & 1.73 & 0.03 & 0.05 \\
\hline El $(k J / d)$ & 8204 & 65 & 8992 & 87 & $<0.0001$ \\
\hline
\end{tabular}

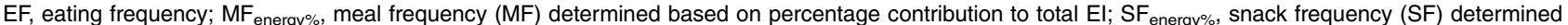
based on percentage contribution to total $\mathrm{El}_{\text {; }} \mathrm{MF}_{\text {self-report }}, \mathrm{MF}$ determined based on self-report; $\mathrm{SF}_{\text {self-report }}$, SF determined based on self-report; MF time $_{\text {, }} \mathrm{MF}$ determined based on the time consumed; $\mathrm{SF}_{\text {time }}$, SF determined based on the time consumed; EI, energy intake.

${ }^{*}$ Analyses are based on participants with complete data on two $24 \mathrm{~h}$ dietary recalls as well as complete information on the variables of interest. All dietary variables are based on mean values of two $24 \mathrm{~h}$ dietary recalls.

$\dagger P$ values for differences between children and adolescents based on independent $t$ test.

$\ddagger$ A meal was defined as any eating episode comprising $\geq 15 \%$ of total El, regardless of the time of day or composition of foods and beverages consumed; all other eating episodes were classified as snacks.

$\S$ Self-reports of breakfast, brunch, lunch, supper and dinner or their equivalents in Spanish were considered as meals; all other self-reported eating events were considered as snacks.

IIMeals were defined as eating events reported during selected times of the day (0.600-09.00, 12.00-14.00 and 17.00-20.00 hours); all other eating occasions were considered as snacks.

regression coefficients (with standard errors) of variation of dietary intakes by one increase of $\mathrm{EF}, \mathrm{MF}$ and SF. Potential confounding factors considered were sex, age, race/ethnicity, family poverty income ratio, education of household head, household size, physical activity, watching television and computer use, weight status, dietary reporting status and survey cycle. We repeated the analyses without adjustment for dietary reporting status as well as those after excluding under- and over-reporters. All reported $P$ values are two-tailed, and $P<0 \cdot 01$ was considered statistically significant to minimize the chance of a type 1 error arising from multiple testing.

\section{Results}

Basic characteristics of the participants are shown in Table 1. The mean value of EF was higher in children than in adolescents. The mean values of MF and SF were also higher in children than in adolescents irrespective of the definition applied, except for no difference in $\mathrm{SF}_{\text {time }}$.

Table 2 shows $\mathrm{EF}, \mathrm{MF}_{\text {self-report }}$ and $\mathrm{SF}_{\text {self-report }}$ (as examples) according to categories of participants' characteristics. In both age groups, there was no sex difference in any measures. For children $\mathrm{EF}$ and $\mathrm{SF}_{\text {self-report }}$ were the highest in non-Hispanic whites and $\mathrm{MF}_{\text {self-report }}$ was the highest in Mexican Americans, with the lowest in non-Hispanic blacks. For adolescents EF was the highest in others and $\mathrm{MF}_{\text {self-report }}$ was the highest in non-Hispanic whites, with the lowest in non-Hispanic blacks. Only in adolescents, there were positive associations between family poverty income ratio and $\mathrm{EF}$ and $\mathrm{MF}_{\text {self-report. Edu- }}$ cation of the household head was positively associated with $\mathrm{MF}_{\text {self-report }}$ in both children and adolescents.
Household size showed an association with $\mathrm{MF}_{\text {self-report }}$ only in adolescents, with the highest value in the '3-4' group and the lowest in the ' $\leq 2$ ' group. All $\mathrm{EF}, \mathrm{MF}_{\text {self-report }}$ and $\mathrm{SF}_{\text {self-report }}$ were positively associated with physical activity in adolescents. Watching television and computer use was inversely associated with $\mathrm{MF}_{\text {self-report }}$ only in children and with EF only in adolescents. $\mathrm{SF}_{\text {self-report was }}$ highest in the ' $\geq 4$ to $<6 \mathrm{~h} / \mathrm{d}$ ' group and lowest in the ' $\geq 6 \mathrm{~h} / \mathrm{d}$ ' group only in adolescents. Weight status was inversely associated with all $\mathrm{EF}, \mathrm{MF}_{\text {self-report }}$ and $\mathrm{SF}_{\text {self-report }}$ in adolescents, while in children $\mathrm{MF}_{\text {self-report }}$ was the highest in those who were underweight and the lowest in those who were overweight. Over-reporters had the highest values for all measures, with under-reporters having the lowest values. In adolescents, survey cycle was associated positively with $\mathrm{MF}_{\text {self-report }}$ and inversely with $\mathrm{SF}_{\text {self-report }}$.

Associations of EF, MF and SF with HEI-2010 and its component intakes are presented in Table 3. Irrespective of the definition of meals, MF was positively associated with HEI-2010 in both age groups. In children, one additional meal per day defined by $\mathrm{MF}_{\text {energy\%, }} \mathrm{MF}_{\text {self-report }}$ and $\mathrm{MF}_{\text {time }}$ increased HEI-2010 by $1.45,3.59$ and 1.72 points, respectively. The corresponding value in adolescents was $1.74,3.56$ and 1.99 points, respectively. All measures of MF also showed positive associations with whole grains. There was a positive association between $\mathrm{MF}_{\text {energy } \%}$ and total fruit in children, while in adolescents $\mathrm{MF}_{\text {energy\% was }}$ associated positively with dairy and inversely with total vegetables, fatty acids ratio and sodium. $\mathrm{MF}_{\text {self-report }}$ was positively associated with total fruit, whole fruit (adolescents only) and dairy, and inversely with the fatty acids ratio (adolescents only) and empty calories. $\mathrm{MF}_{\text {time }}$ showed positive associations with total fruit, whole fruit, 
Table 2 Eating frequency, meal frequency and snack frequency according to categories of participants' characteristics: US children and adolescents, National Health and Nutrition Examination Survey 2003-2012*

\begin{tabular}{|c|c|c|c|c|c|c|c|c|c|c|c|c|c|c|}
\hline & \multicolumn{7}{|c|}{ Children aged $6-11$ years $(n$ 4269) } & \multicolumn{7}{|c|}{ Adolescents aged $12-19$ years ( $n$ 6193) } \\
\hline & \multirow[b]{2}{*}{$n$} & \multicolumn{2}{|c|}{$\begin{array}{c}\mathrm{EF} \\
\text { (times/d) }\end{array}$} & \multicolumn{2}{|c|}{$\begin{array}{l}\mathrm{MF}_{\text {self-report }} \\
\text { (times/d) } \dagger\end{array}$} & \multicolumn{2}{|c|}{$\begin{array}{l}\mathrm{SF}_{\text {self-report }} \\
\text { (times/d) } \dagger\end{array}$} & \multirow[b]{2}{*}{$n$} & \multicolumn{2}{|c|}{$\begin{array}{c}\mathrm{EF} \\
\text { (times/d) }\end{array}$} & \multicolumn{2}{|c|}{$\begin{array}{l}\mathrm{MF}_{\text {self-report }} \\
\text { (times/d) } \dagger\end{array}$} & \multicolumn{2}{|c|}{$\begin{array}{l}\mathrm{SF}_{\text {self-report }} \\
\text { (times/d) }\end{array}$} \\
\hline & & Mean & SE & Mean & SE & Mean & SE & & Mean & SE & Mean & SE & Mean & SE \\
\hline \multicolumn{15}{|l|}{ Sex } \\
\hline $\begin{array}{l}\text { Boys } \\
\text { Girls }\end{array}$ & $\begin{array}{l}2112 \\
2157\end{array}$ & $\begin{array}{l}4.83 \\
4.70\end{array}$ & $\begin{array}{l}0.04 \\
0.04\end{array}$ & $\begin{array}{l}2.89 \\
2.86\end{array}$ & $\begin{array}{l}0.02 \\
0.02\end{array}$ & $\begin{array}{l}1.94 \\
1.83\end{array}$ & $\begin{array}{l}0.04 \\
0.03\end{array}$ & $\begin{array}{l}3162 \\
3031\end{array}$ & $\begin{array}{l}4.20 \\
4.06\end{array}$ & $\begin{array}{l}0.04 \\
0.04\end{array}$ & $\begin{array}{l}2.57 \\
2.52\end{array}$ & $\begin{array}{l}0.02 \\
0.02\end{array}$ & $\begin{array}{l}1.63 \\
1.54\end{array}$ & $\begin{array}{l}0.04 \\
0.03\end{array}$ \\
\hline$P \ddagger$ & & \multicolumn{2}{|c|}{0.013} & \multicolumn{2}{|c|}{0.14} & & & & 0.0 & 12 & 0.6 & & 0.0 & \\
\hline Race/ethnicity & & & & & & & & & & & & & & \\
\hline Non-Hispanic white & 1269 & 4.85 & 0.04 & $2 \cdot 89$ & 0.02 & 1.96 & 0.04 & 1774 & $4 \cdot 18$ & 0.04 & 2.59 & 0.02 & 1.60 & 0.04 \\
\hline Non-Hispanic black & 1137 & 4.37 & 0.04 & $2 \cdot 75$ & 0.03 & 1.62 & 0.04 & 1932 & 3.86 & 0.05 & 2.33 & 0.03 & 1.53 & 0.04 \\
\hline Mexican American & 1164 & 4.77 & 0.05 & 2.96 & 0.03 & 1.81 & 0.04 & 1662 & $4 \cdot 10$ & 0.05 & 2.58 & 0.03 & 1.51 & 0.04 \\
\hline Others & 699 & 4.81 & 0.07 & $2 \cdot 89$ & 0.02 & 1.92 & 0.07 & 825 & 4.22 & 0.07 & 2.54 & 0.04 & 1.68 & 0.06 \\
\hline$P \ddagger$ & & $<0 . c$ & 001 & $<0 \cdot c$ & 01 & $<0$ & 001 & & $<0.0$ & 001 & 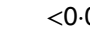 & 001 & 0 . & \\
\hline Family poverty income ratic & & & & & & & & & & & & & & \\
\hline$<130 \%$ & 1855 & 4.69 & 0.05 & 2.87 & 0.02 & 1.82 & 0.04 & 2513 & 4.06 & 0.04 & $2 \cdot 50$ & 0.03 & 1.56 & 0.03 \\
\hline $130-349 \%$ & 1512 & 4.76 & 0.06 & $2 \cdot 87$ & 0.03 & 1.89 & 0.05 & 2259 & 4.04 & 0.04 & $2 \cdot 50$ & 0.02 & 1.55 & 0.04 \\
\hline$\geq 350 \%$ & 902 & 4.85 & 0.05 & $2 \cdot 89$ & 0.02 & 1.96 & 0.06 & 1421 & 4.28 & 0.05 & $2 \cdot 64$ & 0.02 & 1.65 & 0.05 \\
\hline $\bar{P} \ddagger$ & & 0.6 & & 0. & & & & & $<0.0$ & 001 & $<0 \cdot($ & & 0 & \\
\hline Education of household he & & & & & & & & & & & & & & \\
\hline$<12$ years & 1207 & 4.61 & 0.05 & $2 \cdot 81$ & 0.03 & 1.80 & 0.05 & 1871 & 4.09 & 0.05 & $2 \cdot 51$ & 0.03 & 1.58 & 0.04 \\
\hline 12 years & 1035 & 4.76 & 0.06 & $2 \cdot 83$ & 0.03 & 1.93 & 0.05 & 1435 & 4.00 & 0.0 & $2 \cdot 48$ & 0. & 1.52 & 0.05 \\
\hline Some college & 1196 & 4.83 & 0.05 & $2 \cdot 90$ & 0.02 & 1.93 & 0.05 & 1831 & $4 \cdot 15$ & 0.04 & 2.52 & 0.02 & 1.63 & 0.04 \\
\hline College degree or more & 831 & 4.81 & 0.06 & 2.93 & 0.02 & 1.88 & 0.05 & 1056 & $4 \cdot 26$ & 0.07 & $2 \cdot 67$ & 0.03 & 1.59 & 0.06 \\
\hline$P \ddagger$ & & 0.0 & & 0.0 & & 0 & & & 0.0 & & $<0 \cdot($ & & 0 & \\
\hline Household size & & & & & & & & & & & & & & \\
\hline$\leq 2$ & 155 & 4.86 & 0.12 & $2 \cdot 88$ & 0.04 & 1.99 & 0.11 & 495 & 3.93 & 0.08 & $2 \cdot 38$ & 0.05 & 1.56 & 0.07 \\
\hline $3-4$ & 1825 & 4.75 & 0.04 & $2 \cdot 8$ & 0.02 & 1. & 0.04 & 2831 & $4 \cdot 16$ & 0.04 & $2 \cdot 58$ & 0.02 & 1.59 & 0.04 \\
\hline$\geq 5$ & 2289 & 4.77 & 0.04 & $2 \cdot 89$ & 0.01 & 1.88 & 0.04 & 2867 & 4.13 & 0.04 & 2.54 & 0.02 & 1.59 & 0.04 \\
\hline $\bar{P} \ddagger$ & & 0.6 & & 0 & & & & & $0 . c$ & & 0.0 & & 0 & \\
\hline Physical activity§ & & & & & & & & & & & & & & \\
\hline Low & 714 & 4.73 & 0.08 & $2 \cdot 82$ & 0.02 & 1.91 & 0.07 & 972 & 3.86 & 0.05 & $2 \cdot 40$ & 0.04 & 1.46 & 0.04 \\
\hline Mode & 989 & 4.69 & 0.05 & 2.90 & 0.02 & 1.79 & 0.05 & 251 & 4.07 & $0 . c$ & 2.56 & 0. & 1.51 & 0.03 \\
\hline Active & 2566 & 4.80 & 0.03 & $2 \cdot 88$ & 0.02 & 1.92 & 0.04 & 2707 & $4 \cdot 28$ & 0.04 & 2.58 & 0.02 & 1.69 & 0.04 \\
\hline$P \ddagger$ & & 0. & & $0 \cdot($ & & & & & $<0.0$ & 001 & 0.0 & & $<0 \cdot c$ & \\
\hline Watching television and co & puter us & & & & & & & & & & & & & \\
\hline$<2 \mathrm{~h} / \mathrm{d}$ & 1108 & 4.70 & 0.06 & 2.93 & 0.02 & 1.78 & 0.05 & 1097 & $4 \cdot 19$ & 0.07 & $2 \cdot 57$ & 0.03 & 1.62 & 0.06 \\
\hline$\geq 2$ to $<4 \mathrm{~h} / \mathrm{d}$ & 1792 & 4.79 & 0.05 & $2 \cdot 89$ & 0.02 & 1.90 & 0.05 & 1627 & $4 \cdot 19$ & 0.05 & $2 \cdot 57$ & 0.03 & 1.62 & 0.05 \\
\hline$<6 \mathrm{~h} / \mathrm{d}$ & 012 & 4.80 & 0.06 & $2 \cdot 84$ & 0.03 & 1 . & 0.05 & 13 & $4 \cdot 20$ & & $2 \cdot 51$ & & & 0.05 \\
\hline$\geq 6 \mathrm{~h} / \mathrm{c}$ & 526 & 4.75 & 0.09 & 2.77 & 0.04 & 1.99 & 0.08 & 2130 & 4.03 & 0.04 & 2.54 & 0.02 & 1.49 & 0.04 \\
\hline $\bar{P} \ddagger$ & & 0.5 & & 0.0 & & & & & 0.0 & & 0. & & 0.0 & \\
\hline Weight status\| & & & & & & & & & & & & & & \\
\hline Underweight & 122 & 4.76 & 0.12 & 3.00 & 0.05 & 1.76 & 0.13 & 201 & 4.52 & 0.13 & 2.72 & 0.06 & 1.79 & 0.11 \\
\hline Norm & 2595 & 4.81 & 0.04 & 2.91 & 0.01 & 1.90 & 0.03 & 3699 & 4.25 & 0.03 & 2.58 & 0.02 & 1.67 & 0.03 \\
\hline Overweight & 684 & 4.68 & 0.07 & 2.78 & 0.03 & 1.90 & 0.06 & 10 & 3.88 & 0.07 & 2.48 & 0.03 & 1.40 & 0.06 \\
\hline Obes & 868 & 4.67 & 0.05 & $2 \cdot 82$ & 0.03 & 1.86 & 0.05 & 1276 & 3.87 & 0.05 & 2.46 & 0.03 & 1.41 & 0.04 \\
\hline$P \ddagger$ & & 0.6 & & 促 & & & & & $<0$. & & $<0$ & & $<0 . c$ & \\
\hline Dietary reporting status $\mathbb{f}$ & & & & & & & & & & & & & & \\
\hline Under-reporting & 447 & 3.90 & 0.07 & $2 \cdot 50$ & 0.05 & 1.40 & 0.05 & 21 & 3.54 & 0.04 & 2.37 & 0.03 & $1 \cdot 18$ & 0.03 \\
\hline Acceptable reporting & 3425 & 4.77 & 0.03 & $2 \cdot 90$ & 0.01 & 1.86 & 0.03 & 3752 & 4.36 & 0.03 & 2.62 & 0.02 & 1.74 & 0.03 \\
\hline Over-reporting & 397 & 5.53 & 0.10 & 2.99 & 0.03 & 2.54 & 0.09 & 326 & $5 \cdot 10$ & 0.14 & 2.72 & 0.08 & 2.38 & 0.15 \\
\hline $\begin{aligned} P \ddagger \\
\text { Surve }\end{aligned}$ & & $<0 \cdot c$ & 21 & $<0$. & & & & & $<0$ & & & & & \\
\hline Survey cycle & & & & & & & & & & & & & & \\
\hline $2003-2004$ & 775 & 4.82 & 0.06 & $2 \cdot 80$ & 0.03 & 2.02 & 0.05 & 1758 & 4.22 & 0.07 & 2.47 & 0.04 & 1.75 & 0.06 \\
\hline 2005 & 873 & 4.76 & 0.06 & $2 \cdot 86$ & 0.04 & 1.90 & 0.07 & 1716 & $4 \cdot 19$ & 0.06 & 2.53 & 0.03 & 1.66 & 0.06 \\
\hline $2007-2008$ & 813 & 4.74 & 0.06 & $2 \cdot 92$ & 0.02 & 1.83 & 0.05 & 857 & 4.07 & 0.07 & 2.53 & 0.03 & 1.55 & 0.07 \\
\hline $2009-2010$ & 867 & 4.73 & 0.05 & $2 \cdot 88$ & 0.02 & 1.85 & 0.04 & 944 & 4.09 & 0.06 & 2.59 & 0.02 & 1.50 & 0.05 \\
\hline $2011-2012$ & 941 & 4.76 & 0.08 & $2 \cdot 92$ & 0.02 & 1.84 & 0.09 & 918 & 4.08 & 0.06 & $2 \cdot 61$ & 0.03 & 1.46 & 0.06 \\
\hline$P \ddagger$ & & 0 & & 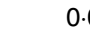 & & & & & 0.4 & & 0.0 & & 0.0 & \\
\hline
\end{tabular}

$\mathrm{EF}$, eating frequency; $\mathrm{MF}_{\text {self-report }}$, meal frequency determined based on self-report; $\mathrm{SF}_{\text {self-report }}$, snack frequency determined based on self-report.

${ }^{*}$ Analyses are based on participants with complete data on two $24 \mathrm{~h}$ dietary recalls as well as complete information on the variables of interest. All dietary variables are based on mean values of two $24 \mathrm{~h}$ dietary recalls.

†Self-reports of breakfast, brunch, lunch, supper and dinner or their equivalents in Spanish were considered as meals; all other self-reported eating events were considered as snacks.

¥Based on Wald's $F$ test.

§The physical activity variable was created as follows. For children aged 6-11 years, the response to the question on the number of times per week that play or exercise was hard enough to induce sweat in the past 7 d (NHANES 2009-2012) or without a specified period (NHANES 2003-2008): 0-3 times = low; 4-6 times = moderate; and 7 times $=$ active. For adolescents aged 12-19 years, the responses to two different questions on any leisure-time moderate or vigorous activities lasting $\geq 10 \mathrm{~min}$ in the past $30 \mathrm{~d}$ (NHANES 2003-2006) or without a specified period (NHANES 2007-2012): no to both two questions = low; yes to one question = moderate; and yes to both two questions $=$ active.

IIDefined based on the percentile of BMI-for-age ${ }^{(50)}$ : $<5$ th percentile for underweight; $\geq 5$ th to $<85$ th percentile for normal; $\geq 85$ th to $<95$ th percentile for overweight; and $\geq 95$ th percentile for obese.

IDefined based on ratio of reported energy intake to estimated energy requirement: $<0.69$ for under-reporting; $0.69-1.46$ for acceptable reporting; and $>1.46$ for over-reporting. 


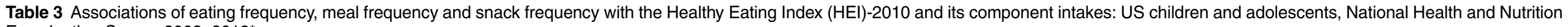
Examination Survey 2003-2012*

\begin{tabular}{|c|c|c|c|c|c|c|c|c|c|c|c|c|c|c|c|c|c|c|c|c|c|c|c|}
\hline & \multirow[b]{2}{*}{ Mean } & \multirow[b]{2}{*}{ SE } & \multicolumn{3}{|c|}{ EF (times/d) } & \multicolumn{3}{|c|}{$\mathrm{MF}_{\text {energy\% }}$ (times/d) $\dagger$} & \multicolumn{3}{|c|}{$\mathrm{SF}_{\text {energy\% }}$ (times/d) $\dagger$} & \multicolumn{3}{|c|}{$\mathrm{MF}_{\text {self-report }}($ times $/ \mathrm{d}) \ddagger$} & \multicolumn{3}{|c|}{$\mathrm{SF}_{\text {self-report }}($ times $/ \mathrm{d}) \ddagger$} & \multicolumn{3}{|c|}{$\mathrm{MF}_{\text {time }}($ times $/ \mathrm{d}) \S$} & \multicolumn{3}{|c|}{$\mathrm{SF}_{\text {time }}($ times $/ \mathrm{d}) \S$} \\
\hline & & & $\beta \|$ & SEll & $P$ & $\beta \|$ & SEll & $P$ & $\beta \|$ & SEll & $P$ & $\beta \|$ & SEll & $P$ & $\beta \|$ & SEll & $P$ & $\beta \|$ & sell & $P$ & $\beta \|$ & SEll & $P$ \\
\hline \multicolumn{24}{|l|}{ Children aged 6} \\
\hline $\mathrm{HEI}-2 \mathrm{C}$ & 47.0 & 34 & $1 \cdot 21$ & 0.25 & 0.0001 & 1.45 & 0.50 & 0.005 & 0.70 & 0.21 & 0.001 & 3.59 & 0.58 & $<0.0001$ & 0.60 & 0.27 & 0.03 & 1.72 & 0.32 & $<0.0001$ & 0.10 & 0.26 & 0.70 \\
\hline Total $\mathrm{ff}$ & 0.62 & תר & 0.09 & 0.01 & 0.0001 & 0.07 & 0.02 & 004 & 0.06 & 0.01 & $<0.0001$ & $\cdot 12$ & 0.03 & 0.0002 & 0.07 & 0.01 & $<0.0001$ & 0.10 & 0.01 & $<0.0001$ & 0.03 & 0.01 & 0.04 \\
\hline Whole & 0.34 & 0.01 & 0.04 & 0.01 & $<0.0001$ & 0.03 & 0.02 & 0.13 & 0.03 & 0.01 & 0.0002 & 05 & 0.03 & 0.05 & 0.04 & 0.01 & $<0.0001$ & 0.05 & 0.01 & $<0.0001$ & 0.01 & 01 & 0.26 \\
\hline $\begin{array}{l}\text { Total vegetables } \\
\quad(\text { cup eq. } / 4184 \mathrm{~kJ})\end{array}$ & 0.51 & 0.01 & -0.02 & 0.01 & 0.011 & -0.02 & 0.02 & 0.17 & -0.01 & 0.01 & 0.09 & 0.02 & 0.02 & 0.32 & -0.03 & 0.01 & 0.003 & -0.01 & 0.01 & 0.50 & -0.02 & 0.01 & 0.011 \\
\hline $\begin{array}{l}\text { Greens and beans } \\
\text { (cup eq./4184 kJ) }\end{array}$ & 0.043 & 0.002 & -0.002 & 0.002 & 0.30 & -0.001 & 0.005 & 0.75 & -0.002 & 0.002 & 0.47 & 0.003 & 0.006 & 0.67 & -0.003 & 0.003 & 0.22 & 0.000 & 0.002 & 0.84 & -0.004 & 0.003 & 0.20 \\
\hline $\begin{array}{l}\text { Whole grains } \\
\text { (oz eq. } / 4184 \mathrm{~kJ})\end{array}$ & 0.35 & 0.01 & 0.03 & 0.01 & 0.004 & 0.10 & 0.02 & $<0.0001$ & 0.004 & 0.010 & 0.70 & $0 \cdot 10$ & 0.02 & $<0.0001$ & 0.01 & 0.01 & 0.27 & 0.05 & 0.01 & $<0.0001$ & -0.005 & 0.011 & 0.64 \\
\hline Dairy ( & $1 \cdot 18$ & 0.02 & 0.03 & 0.01 & 0.0 & 0.05 & 0.03 & 0.05 & 0.01 & 0.01 & 0.3 & 14 & 0.02 & $<0 . c$ & 0.002 & 0.013 & 0. & 0.02 & 0.02 & 0.19 & 0.01 & 0.01 & 0.28 \\
\hline $\begin{array}{l}\text { Total protein foods } \\
(\mathrm{oz} \text { eq. } / 4184 \mathrm{~kJ}\end{array}$ & $2 \cdot 21$ & 0.03 & -0.14 & 0.02 & $<0.0$ & -0.05 & 0.0 & 0.35 & -0. & 0.02 & $<0.0001$ & 01 & & & 0 & 0.02 & $<0$ & -0.10 & & 0.009 & $-0 \cdot 10$ & 0.03 & 0.0004 \\
\hline $\begin{array}{l}\text { Seafood and plant proteins } \\
\text { (oz eq./4184 kJ) }\end{array}$ & 0.40 & 0.02 & -0.05 & 0.03 & 0.08 & -0.02 & 0.05 & 0.71 & -0.03 & 0.02 & 0.06 & 0.02 & 0.03 & 0.44 & -0.05 & 0.03 & 0.06 & -0.04 & 0.03 & 0.20 & -0.02 & 0.02 & 0.20 \\
\hline Fatty a & 1.70 & 0.01 & -0.02 & 0.01 & 0.09 & -0.04 & 0.02 & 0.09 & -0.01 & 0.0 & 0.30 & -0.06 & 0.03 & 0.0 & -0.01 & 0.0 & 0.3 & -0.03 & 0.01 & 0.05 & -0.01 & 01 & 0.56 \\
\hline $\begin{array}{r}\text { Refined } \\
\text { (oz e }\end{array}$ & 3.09 & 0.03 & -0.08 & 0.0 & .005 & $\lambda$ & 0.0 & $5 /$ & 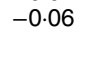 & 0.0 & 0.0 & $0 . c$ & & r & 0 & 0. & 0. & 0 & 0 & 0.1 & -0.06 & 0.03 & 0.02 \\
\hline Sodiun & 1.57 & 0.01 & -0.06 & 0.01 & $<0.0001$ & -0.03 & 0.01 & 0.04 & -0.04 & 0.01 & 0.0001 & -0.01 & 0.02 & 0.7 & -0.06 & 0.01 & $<0.0001$ & -0.04 & 0.01 & $<0.0001$ & -0.04 & 0.01 & $<0.0001$ \\
\hline $\begin{array}{l}\text { Empty calories } \\
(\% \text { of energy })^{\star *}\end{array}$ & 33.5 & 0.2 & 0.005 & 0.177 & & 0 & & 0.33 & 0.09 & 0.1 & & 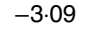 & & 01 & & 0.18 & & -0.77 & 0 & 0. & 0.67 & ).21 & 0.002 \\
\hline \multicolumn{24}{|c|}{ Adolescents aged $12-19$ years ( $n$ 6193) } \\
\hline HEI-2010 & 43.9 & 0.27 & 1.52 & 0.20 & 0.0 & 1.74 & 0.37 & $<0.0001$ & 1.00 & 0.2 & 1 & 3.56 & 0. & $<0 . c$ & 0. & 0.2 & & 1. & 0. & $<0$ & 0.6 & 0.25 & 0.77 \\
\hline & 0.46 & 0.02 & 0.07 & 0.01 & $<$ & 0.05 & 0.02 & & 0.05 & 0.0 & & & 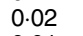 & & & & & & & & & & \\
\hline Whole & 0.21 & 0.0 & 0.04 & 0.0 & $<0.0$ & 0 . & 0 & 0.6 & 0. & $0 \cdot($ & 01 & 0.05 & 0 . & $<0$ & 0 & 0.0 & $<0$ & 0 & 0 & $<0$ & 0.01 & 0.01 & 0.38 \\
\hline $\begin{array}{l}\text { Total vegetables } \\
\quad(\text { cup eq. } / 4184 \mathrm{~kJ})\end{array}$ & 0.59 & 0.01 & -0.0 & 0.0 & $0 . c$ & -0.04 & 0 & 0.005 & -0 & 0.01 & 0. & 0.03 & 0 & 0 & -0. & 0.0 & 0.0004 & -0.01 & 0. & & -0.02 & 0.01 & 0.05 \\
\hline $\begin{array}{l}\text { Greens and beans } \\
\text { (cup eq./4184 kJ) }\end{array}$ & 0.046 & 0.003 & -0.003 & 0.002 & 0.23 & -0.003 & 0.004 & 0.41 & -0.002 & 0.002 & 0.43 & 0.005 & 0.004 & 0.28 & -0.005 & 0.002 & 0.04 & 0.003 & 0.002 & 0.30 & -0.006 & 0.003 & 0.03 \\
\hline $\begin{array}{l}\text { Whole grains } \\
\text { (oz eq. } / 4184-\mathrm{kJ})\end{array}$ & 0.30 & 0.01 & 0.05 & 0.01 & 0.0004 & 0.06 & 0.02 & 0.003 & 0.03 & 0.01 & 0.02 & 0.08 & 0.01 & $<0.0001$ & 0.02 & 0.01 & 0.08 & 0.04 & 0.01 & 0.0005 & 0.02 & 0.02 & 0.13 \\
\hline $84 \mathrm{~kJ})$ & $1 \cdot 01$ & 0.0 & 0.0 & 0.0 & & 0.11 & 0 & 1 & -0.6 & 0.0 & & 018 & & & -0 & 001 & & 0.05 & 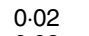 & & -0 & 0.01 & \\
\hline $\begin{array}{l}\text { Total pi } \\
(\mathrm{oz} \in\end{array}$ & 0 & 0 & -0.14 & 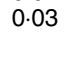 & 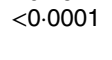 & - & 0.0 & 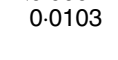 & - & $0 . c$ & & 0.02 & 0.05 & & . & 0.03 & & 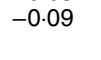 & 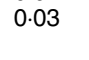 & & -0 & 0.03 & 3 \\
\hline $\begin{array}{l}\text { Seafood and plant proteins } \\
\text { (oz eq./4184 kJ) }\end{array}$ & 0.37 & 0.02 & 0.04 & 0.01 & 0.004 & 0.02 & 0.03 & 0.44 & 0.03 & 0.01 & 0.006 & 0.01 & 0.03 & 0.6 & 0.04 & 0.02 & 0.02 & 0.04 & 0.01 & .007 & 0.01 & 0.01 & 0.29 \\
\hline Fatty acids $\mathbf{q}$ & 1.80 & 0.01 & -0.04 & 0.01 & 0.0006 & -0.10 & 0.02 & $<0.0001$ & -0.01 & 0.0 & 0.36 & -0.1 & 0.02 & & & 0.011 & & -0.04 & 0.01 & 0.002 & -0 & 0.0 & 0. \\
\hline $\begin{array}{l}\text { Refined grains } \\
\text { (oz eq. } / 4184 \mathrm{~kJ})\end{array}$ & 3.16 & 0.02 & 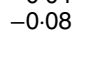 & 0.0 & & 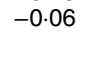 & 0 & & 0 & 0.0 & $0 .($ & 0.0 & 0. & & & 0.02 & & 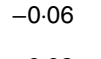 & 0 & & -0.04 & $0 \cdot($ & \\
\hline Sodium $(\mathrm{g} / 4184 \mathrm{~kJ})$ & 1.66 & 0.01 & -0.05 & 0.01 & $<0 \cdot$ & -0.0 & 0. & & -0.0 & 0.0 & $<0$ & 0.04 & 0 & & -0.0 & $0 . c$ & & -0.03 & 0 & & -0.04 & 0.0 & \\
\hline $\begin{array}{l}\text { Empty calories } \\
\left(\% \text { of energy) }{ }^{\star \star}\right.\end{array}$ & 6 & 0 & 0.35 & 20 & & 0 & 0 & & 0 & 0. & & & 0.36 & & 1 & 0.18 & 0001 & -0.29 & 0.23 & & 0.72 & 0.22 & 0.001 \\
\hline
\end{tabular}

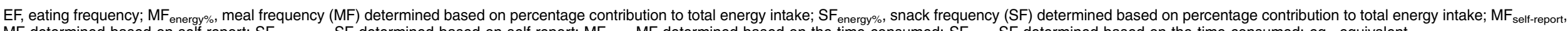
$\mathrm{MF}$ determined based on self-report; $\mathrm{SF}_{\text {self-report, }} \mathrm{SF}$ determined based on self-report; $\mathrm{MF}_{\text {time, }}$, MF determined based on the time consumed; $\mathrm{SF}$ time, $\mathrm{SF}$ determined based on the time consumed; eq., equivalent.

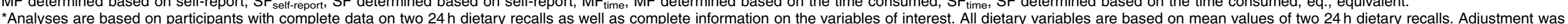

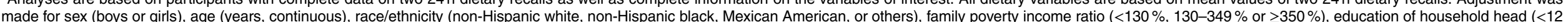

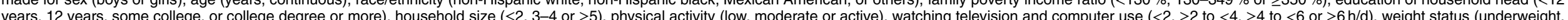
normal, overweight or obese), dietary reporting status (under-reporting, acceptable reporting or over-reporting) and survey cycle (2003-2004, 2005-2006, 2007-2008, 2009-2010 or 2011-2012).

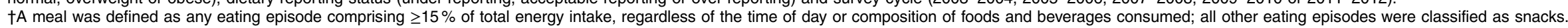

\#Self-reports of breakfast, brunch, lunch, supper and dinner or their equivalents in Spanish were considered as meals; all other self-reported eating events were considered as snacks.

$\S$ Meals were defined as eating events reported during selected times of the day (0.600-09.00, 12.00-14.00 and 17.00-20.00 hours); all other eating occasions were considered as snacks.

IIRegression coefficients mean the change of dietary variables with one additional eating occasion per day.

${ }_{\star \star \star}$ Energy from solid fats, alcohol and added sugars. 
dairy (adolescents only), and seafood and plant proteins (adolescents only), and inverse associations with total protein foods, fatty acids ratio (adolescents only), sodium (children only) and empty calories (children only).

The associations for SF varied depending on the definition of snacks. While $\mathrm{SF}_{\text {energy\% }}$ was positively associated with HEI-2010 in both children and adolescents (0.70 and 1.00 point increase by one additional snack, respectively), $\mathrm{SF}_{\text {self-report }}$ and $\mathrm{SF}_{\text {time }}$ showed no associations. $\mathrm{SF}_{\text {energy\% }}$ was associated positively with total fruit, whole fruit, and seafood and plant proteins (adolescents only), and inversely with total protein foods and sodium. $\mathrm{SF}_{\text {self-report }}$ showed positive associations with total fruit, whole fruit and empty calories, and inverse associations with total vegetables, dairy (adolescents only), total protein foods, refined grains and sodium. $\mathrm{SF}_{\text {time }}$ was positively associated with empty calories and inversely with total protein foods and sodium. EF was positively associated with HEI-2010, total fruit, whole fruit, whole grains, and seafood and plant proteins (adolescents only), and inversely with total protein foods, fatty acids ratio (adolescents only), refined grains and sodium.

All analyses were conducted without adjustment for dietary reporting status (data not shown) or after excluding under- and over-reporters ( $n 447$ and 397 in children and 2115 and 326 in adolescents, respectively; see online supplementary material, Supplemental Table 1), providing similar results.

\section{Discussion}

To our knowledge, the present study is the first to examine associations of different measures of MF and SF with diet quality, after taking into account the confounding of EI misreporting. In a representative sample of US children and adolescents, higher MF was associated with higher diet quality as assessed by HEI-2010. This was not dependent on the definition of meals. The associations were not confounded by misreporting of EI, as the results did not change before and after adjustment for EI misreporting and when only those with plausible EI were analysed. However, the associations for SF varied depending on the definition of snacks. While SF based on energy contribution was positively associated with diet quality, there were no associations for SF based on self-report or based on time. Thus, the study suggests the importance of the use of different definition of meals and snacks to better understand the effects of different dietary patterns on health.

While many epidemiological studies which have investigated the association between $\mathrm{EF}$ (i.e. sum of $\mathrm{MF}$ and $\mathrm{SF}$ ) and dietary intakes have yielded inconsistent outcomes $^{(8-14)}$, there have been few attempts to investigate the effects of MF and SF separately. In Swedish adults, SF assessed by a questionnaire showed positive associations with intakes of confectionery, fat, sugar and alcohol, and inverse associations with intakes of protein and dietary fibre ${ }^{(11)}$. In contrast, SF calculated based on self-report was associated with higher intakes of vitamins $\mathrm{A}, \mathrm{C}$ and $\mathrm{E}$, $\beta$-carotene, $\mathrm{Mg}$ and $\mathrm{K}$ in US elderly people ${ }^{(12)}$. SF calculated based on self-report was also associated with a higher score for HEI-2005 in US adults ${ }^{(13)}$. A higher MF was associated with higher diet quality as assessed by the Canadian HEI among Canadian elderly people, although no clear definition of meal was provided ${ }^{(54)}$. A previous US study showed that SF calculated based on self-report, but not MF, was positively associated with diet quality (as assessed by the HEI-2005) in children (aged 9-11 years) ${ }^{(7)}$. Conversely, in adolescents (aged 12-15 years) SF was inversely associated with diet quality, while MF showed a positive association $^{(7)}$. In the present study, while MF was associated with better diet quality irrespective of the definition of meals, the associations for SF varied depending on the definition of snacks. These inconsistent observations may be, at least partly, due to differences in the characteristics and lifestyles of the populations, definitions of MF and SF, dietary assessment methods and potential confounding factors considered.

While MF was more strongly associated with diet quality than SF based on any definition of meals and snacks we applied, the difference in the strength of the associations was the smallest when meals and snacks were defined based on EI contribution, suggesting that this definition may not appropriately differentiate meals and snacks in terms of nutritional quality of eating occasions. This may explain why only $\mathrm{SF}_{\text {energy\% }}$ (but not $\mathrm{SF}_{\text {self-report }}$ or $\mathrm{SF}_{\text {time }}$ ) was positively associated with diet quality. Conversely, the difference in the strength of the associations was the largest when meals and snacks were defined based on participants' self-report. Of particular interest is that higher $\mathrm{MF}_{\text {self-report }}$ did not contribute to overconsumption of (unhealthy) foods but rather decreased the consumption of empty calories. Thus the classification of meals and snacks based on self-report, rather than contribution to total EI and time, may be more informative for investigating the effect of MF and SF on dietary intakes, at least in US children and adolescents. However, oversimplification should be avoided because there is no consensus about what constitutes a snack or a meal. For example, MF and SF based on self-report are subject to inconsistencies due to differences in individual perception ${ }^{(31)}$. In accordance with a previous analysis based on $\operatorname{NHANES}^{(44)}$, eating occasions reported as 'extended consumption', 'other' and 'don't know' were considered as snacks, but this may not be appropriate at least for some individuals; further research is needed to clarify the kind and the amount of foods consumed in such eating occasions. Additionally, MF and SF based on time may be problematic because eating patterns vary according to lifestyle (e.g. shift workers, individuals who consistently eat their meals at non-traditional times of day) as well as the cultural environment ${ }^{(31)}$. Furthermore, MF and SF 
based on energy contribution ( $\geq 15 \%$ or $<15 \%$ ) was made on the basis of the US adults' national averages of the distribution of energy from (self-defined) meals compared with (self-defined) snacks (breakfast $\approx 16 \%$; lunch $\approx 25 \%$; dinner $\approx 37 \%$; and snack $\approx 22 \%$ from two occasions) ${ }^{(44)}$, but this may not be suitable in children and adolescents. Thus, results may possibly differ on the basis of other definitions. In any case, as research explicitly examining the impact of these different definitions is limited, further research using different definitions of meals and snacks is warranted.

The strengths of the present study include the use of a variety of published definitions of MF and SF based on detailed dietary information obtained from two $24 \mathrm{~h}$ dietary recalls and the use of individualized measures of EER to assess misreporting of $\mathrm{EI}$ in a large representative sample of US children and adolescents. However, there are also several limitations. First, due to the cross-sectional design of the present study, the assessment of causality cannot be addressed. Considering the advice frequently made by individuals in the health-care professions and in the lay press that those who want to lose weight should eat small, frequent meals ${ }^{(25,55)}$, overweight and obese individuals may increase their MF and EF. Alternatively, overweight and obese individuals may simply decrease their MF, SF and EF in an attempt to lose weight. To minimize the potential effect, we included weight status as a potential confounding factor.

All self-reported dietary assessment methods are subject to both random and systematic measurement errors ${ }^{(56)}$. Given the day-to-day variability in eating patterns of free-living individuals, estimates of dietary intakes and behaviours derived from two $24 \mathrm{~h}$ dietary recalls unlikely represent the usual intakes or behaviours of individual respondents. Nevertheless, as this kind of random error would tend to result in bias towards attenuating rather than enhancing the relationship, more recall days would have provided stronger associations. A recent analysis based on NHANES ${ }^{(57)}$ has shown that misreporting (under- and over-reporting) of EI is prevalent particularly in adolescents aged 12-19 years (32.6\% and $4.8 \%$, respectively), although the prevalence of under- and overreporting in children aged 6-11 years (8.7\% and 9.6\%, respectively) was not high (this may, at least partly, be due to the parental help in the recall $\left.{ }^{(58)}\right)$. This kind of bias may distort true relationships between dietary behaviours and intakes or even create spurious ones. However, we believe that the associations observed here were not seriously influenced by misreporting of EI, because the results did not change before and after adjustment for EI misreporting and when only participants with plausible EI were analysed.

At present, the only way to obtain unbiased information on energy requirements in free-living settings is to use doubly labelled water ${ }^{(56)}$. This technique is expensive and impractical for application in large-scale epidemiological studies. Instead, in the present study, EER was calculated using equations from the US Dietary Reference Intakes, which have been developed based on a large number of measurements of total energy expenditure by the doubly labelled water method and are highly accurate $\left(R^{2} \geq 0.95\right)^{(51)}$. In the absence of actual, measured total energy expenditure, these equations should serve as the best proxy. Because of constraints within the data set, we did not have a validated and individualized measure of physical activity. Although some measures of self-reported physical activity were available, we decided not to use such variables in the present study because self-report of physical activity suffers from significant reporting bias mainly attributable to a combination of social desirability bias and the cognitive challenge associated with estimating frequency and duration of physical activity, particularly for children and adolescents ${ }^{(59)}$. Instead, we assumed 'low active' level of physical activity for all participants during the calculation of EER. This seems adequate for most US children and adolescents, based on accelerometer-based data in NHANES 2003-2006 ${ }^{(59,60)}$. Nevertheless, in very active individuals (e.g. those aged 6-11 years, as has been reported $)^{(60)}$, EER would be underestimated, resulting in an overestimation of EI:EER. Further, although we adjusted for a variety of potential confounding variables, residual confounding could not be ruled out. Finally, in view of the multiple analyses, it is possible that some of the findings in the present study occurred by chance.

\section{Conclusion}

In the present cross-sectional study in a representative sample of US children and adolescents based on NHANES 2003-2012, we showed positive associations between MF and diet quality as assessed by HEI-2010. These associations were observed whatever definition of meals, i.e. based on EI contribution, based on self-report or based on time, was applied and was not confounded by EI misreporting. In contrast, the associations for $\mathrm{SF}$ varied depending on the definition of snacks. While SF based on energy contribution was positively associated with diet quality, SF based on self-report or based on time showed no associations. In any case, replacing snack occasions to meal occasions may be a good strategy for improving diet quality. Future studies should divide EF into MF and SF based on a variety of definitions of meals and snacks so that any effects of different dietary patterns on health can be properly investigated.

\section{Acknowledgements}

Financial support: This work was supported in part by the Grants-in-Aid for Young Scientists (B) from the Ministry of Education, Culture, Sports, Science and Technology of Japan (K.M., grant number 15K16213). The Ministry of 
Education, Culture, Sports, Science and Technology of Japan had no role in the design, analysis or writing of this article. Conflict of interest: None. Authorship: K.M. contributed to the concept and design of the study, statistical analysis, data interpretation and manuscript writing. M.B.E.L. critically reviewed the manuscript. Both authors read and approved the final manuscript. Ethics of buman subject participation: This study was conducted according to the guidelines laid down in the Declaration of Helsinki and all procedures involving human subjects were approved by National Center for Health Statistics Research Ethics Review Board. Written informed consent was obtained from all participants or proxies.

\section{Supplementary material}

To view supplementary material for this article, please visit http://dx.doi.org/10.1017/S1368980016000069

\section{References}

1. Dietz WH (1994) Critical periods in childhood for the development of obesity. Am J Clin Nutr 59, 955-959.

2. McGill HC (1997) Childhood nutrition and adult cardiovascular disease. Nutr Rev 55, 1 Pt 2, S2-S8.

3. te Velde SJ, Twisk JWR \& Brug J (2007) Tracking of fruit and vegetable consumption from adolescence into adulthood and its longitudinal association with overweight. $\mathrm{Br} J \mathrm{Nutr}$ 98, 431-438.

4. Bertheke Post G, de Vente W, Kemper HC et al. (2001) Longitudinal trends in and tracking of energy and nutrient intake over 20 years in a Dutch cohort of men and women between 13 and 33 years of age: the Amsterdam growth and health longitudinal study. Br J Nutr 85, 375-385.

5. Popkin BM \& Duffey KJ (2010) Does hunger and satiety drive eating anymore? Increasing eating occasions and decreasing time between eating occasions in the United States. Am J Clin Nutr 91, 1342-1347.

6. Fryar CD, Carroll MD \& Ogden CL (2014) Prevalence of overweight and obesity among children and adolescents: United States, 1963-1965 through 2011-2012. NCHS Health E-Stats. http://www.cdc.gov/nchs/data/hestat/obesity_child_ 11_12/obesity_child_11_12.htm (accessed December 2015).

7. Evans EW, Jacques PF, Dallal GE et al. (2015) The role of eating frequency on total energy intake and diet quality in a low-income, racially diverse sample of schoolchildren. Public Health Nutr 18, 474-481.

8. Titan SM, Bingham S, Welch A et al. (2001) Frequency of eating and concentrations of serum cholesterol in the Norfolk population of the European prospective investigation into cancer (EPIC-Norfolk): cross sectional study. BMJ 323, 1286-1288.

9. Holmback I, Ericson U, Gullberg B et al. (2010) A high eating frequency is associated with an overall healthy lifestyle in middle-aged men and women and reduced likelihood of general and central obesity in men. Br J Nutr 104, 1065-1073.

10. Smith KJ, Blizzard L, McNaughton SA et al. (2012) Daily eating frequency and cardiometabolic risk factors in young Australian adults: cross-sectional analyses. Br J Nutr 108, 1086-1094.

11. Berteus Forslund H, Torgerson JS, Sjostrom L et al. (2005) Snacking frequency in relation to energy intake and food choices in obese men and women compared to a reference population. Int J Obes (Lond) 29, 711-719.
12. Zizza CA, Arsiwalla DD \& Ellison KJ (2010) Contribution of snacking to older adults' vitamin, carotenoid, and mineral intakes. J Am Diet Assoc 110, 768-772.

13. Zizza CA \& Xu B (2012) Snacking is associated with overall diet quality among adults. J Acad Nutr Diet 112, 291-296.

14. Leech RM, Worsley A, Timperio A et al. (2015) Understanding meal patterns: definitions, methodology and impact on nutrient intake and diet quality. Nutr Res Rev 28, $1-21$.

15. Marin-Guerrero AC, Gutierrez-Fisac JL, Guallar-Castillon P et al. (2008) Eating behaviours and obesity in the adult population of Spain. Br J Nutr 100, 1142-1148.

16. Berg C, Lappas G, Wolk A et al. (2009) Eating patterns and portion size associated with obesity in a Swedish population. Appetite 52, 21-26.

17. Berteus Forslund H, Lindroos AK, Sjostrom L et al. (2002) Meal patterns and obesity in Swedish women - a simple instrument describing usual meal types, frequency and temporal distribution. Eur J Clin Nutr 56, 740-747.

18. van der Heijden AA, Hu FB, Rimm EB et al. (2007) A prospective study of breakfast consumption and weight gain among US men. Obesity (Silver Spring) 15, 2463-2469.

19. Howarth NC, Huang TTK, Roberts SB et al. (2007) Eating patterns and dietary composition in relation to BMI in younger and older adults. Int J Obes (Lond) 31, 675-684.

20. Drummond SE, Crombie NE, Cursiter MC et al. (1998) Evidence that eating frequency is inversely related to body weight status in male, but not female, non-obese adults reporting valid dietary intakes. Int J Obes Relat Metab Disord 22, 105-112.

21. Ma Y, Bertone ER, Stanek EJ 3rd et al. (2003) Association between eating patterns and obesity in a free-living US adult population. Am J Epidemiol 158, 85-92.

22. Summerbell CD, Moody RC, Shanks J et al. (1996) Relationship between feeding pattern and body mass index in 220 free-living people in four age groups. Eur J Clin Nutr 50, 513-519.

23. Duval K, Strychar I, Cyr MJ et al. (2008) Physical activity is a confounding factor of the relation between eating frequency and body composition. Am J Clin Nutr 88, 1200-1205.

24. Murakami K \& Livingstone MBE (2014) Eating frequency in relation to body mass index and waist circumference in British adults. Int J Obes (Lond) 38, 1200-1206.

25. Hartline-Grafton HL, Rose D, Johnson CC et al. (2010) The influence of weekday eating patterns on energy intake and BMI among female elementary school personnel. Obesity (Silver Spring) 18, 736-742.

26. Mills JP, Perry CD \& Reicks M (2011) Eating frequency is associated with energy intake but not obesity in midlife women. Obesity (Silver Spring) 19, 552-559.

27. Ruidavets JB, Bongard V, Bataille V et al. (2002) Eating frequency and body fatness in middle-aged men. Int J Obes Relat Metab Disord 26, 1476-1483.

28. Kant AK, Schatzkin A, Graubard BI et al. (1995) Frequency of eating occasions and weight change in the NHANES I Epidemiologic Follow-up Study. Int J Obes Relat Metab Disord 19, 468-474.

29. Huang TT, Roberts SB, Howarth NC et al. (2005) Effect of screening out implausible energy intake reports on relationships between diet and BMI. Obes Res 13, 1205-1217.

30. Yannakoulia M, Melistas L, Solomou E et al. (2007) Association of eating frequency with body fatness in preand postmenopausal women. Obesity (Silver Spring) 15, 100-106.

31. Johnson GH \& Anderson GH (2010) Snacking definitions: impact on interpretation of the literature and dietary recommendations. Crit Rev Food Sci Nutr 50, 848-871.

32. Zipf G, Chiappa M, Porter KS et al. (2013) National health and nutrition examination survey: plan and operations, 1999-2010. Vital Health Stat 1 issue 56, 1-37. 
33. Johnson CL, Paulose-Ram R, Ogden CL et al. (2013) National health and nutrition examination survey: analytic guidelines, 1999-2010. Vital Health Stat 2 issue 161, 1-24.

34. Centers for Disease Control and Prevention, National Center for Health Statistics (2015) National Health and Nutrition Examination Survey. NHANES response rates and population totals. http://www.cdc.gov/nchs/nhanes/response_ rates_cps.htm (accessed December 2015).

35. Centers for Disease Control and Prevention, National Center for Health Statistics (2015) National Health and Nutrition Examination Survey. Questionnaires, datasets, and related documentation. http://www.cdc.gov/nchs/nhanes/nhanes_ questionnaires.htm (accessed December 2015).

36. Moshfegh AJ, Rhodes DG, Baer DJ et al. (2008) The US Department of Agriculture Automated Multiple-Pass Method reduces bias in the collection of energy intakes. Am J Clin Nutr 88, 324-332.

37. Blanton CA, Moshfegh AJ, Baer DJ et al. (2006) The USDA Automated Multiple-Pass Method accurately estimates group total energy and nutrient intake. J Nutr 136, 2594-2599.

38. Conway JM, Ingwersen LA, Vinyard BT et al. (2003) Effectiveness of the US Department of Agriculture 5-step multiple-pass method in assessing food intake in obese and nonobese women. Am J Clin Nutr 77, 1171-1178.

39. Conway JM, Ingwersen LA \& Moshfegh AJ (2004) Accuracy of dietary recall using the USDA five-step multiple-pass method in men: an observational validation study. J Am Diet Assoc 104, 595-603.

40. Guenther PM, Casavale KO, Reedy J et al. (2013) Update of the Healthy Eating Index: HEI-2010. J Acad Nutr Diet 113, 569-580.

41. Guenther PM, Kirkpatrick SI, Reedy J et al. (2014) The Healthy Eating Index-2010 is a valid and reliable measure of diet quality according to the 2010 Dietary Guidelines for Americans. J Nutr 144, 399-407.

42. US Department of Agriculture \& US Department of Health and Human Services (2010) Dietary Guidelines for Americans, 2010, 7th ed. Washington, DC: US Government Printing Office; available at http://www.cnpp.usda.gov/ dietaryguidelines.htm

43. US Department of Agriculture, Center for Nutrition Policy and Promotion (2013) Healthy Eating Index support files 07 08. http://www.cnpp.usda.gov/healthy-eatingindex-support-files-07-08 (accessed July 2015).

44. Kant AK \& Graubard BI (2015) 40-Year trends in meal and snack eating behaviors of American adults. J Acad Nutr Diet 115, 50-63.

45. Ritchie LD (2012) Less frequent eating predicts greater BMI and waist circumference in female adolescents. Am J Clin Nutr 95, 290-296.

46. Jennings A, Cassidy A, van Sluijs EM et al. (2012) Associations between eating frequency, adiposity, diet, and activity in 9-10 year old healthy-weight and centrally obese children. Obesity (Silver Spring) 20, 1462-1468.

47. Kant AK \& Graubard BI (2012) Race-ethnic, family income, and education differentials in nutritional and lipid biomarkers in US children and adolescents: NHANES 2003-2006. Am J Clin Nutr 96, 601-612.

48. Kuczmarski RJ, Ogden CL, Guo SS et al. (2002) 2000 CDC Growth Charts for the United States: methods and development. Vital Health Stat 11, issue 246 1-190.

49. Centers for Disease Control and Prevention, National Center for Health Statistics (2015) A SAS program for the 2000 CDC Growth Charts (ages 0 to $<20$ years). http://www.cdc.gov/ nccdphp/dnpao/growthcharts/resources/sas.htm (accessed December 2015).

50. Centers for Disease Control and Prevention (2015) Basics about childhood obesity. http://www.cdc.gov/obesity/ childhood/defining.html (accessed December 2015).

51. Institute of Medicine (2002) Dietary Reference Intakes for Energy, Carbohydrate, Fiber, Fat, Fatty Acids, Cholesterol, Protein and Amino Acids. Washington, DC: National Academy Press.

52. Huang TT, Howarth NC, Lin BH et al. (2004) Energy intake and meal portions: associations with BMI percentile in US children. Obes Res 12, 1875-1885.

53. Centers for Disease Control and Prevention, National Center for Health Statistics (2015) Continuous NHANES web tutorial. http://www.cdc.gov/nchs/tutorials/Nhanes/index_ continuous.htm (accessed December 2015).

54. Shatenstein B, Gauvin L, Keller H et al. (2013) Baseline determinants of global diet quality in older men and women from the NuAge cohort. J Nutr Health Aging 17, 419-425.

55. Mattson MP (2005) The need for controlled studies of the effects of meal frequency on health. Lancet 365, 1978-1980.

56. Livingstone MBE \& Black AE (2003) Markers of the validity of reported energy intake. J Nutr 133, Suppl. 3, 895S-920S.

57. Murakami K \& Livingstone MBE (2015) Prevalence and characteristics of misreporting of energy intake in US children and adolescents: National Health and Nutrition Examination Survey (NHANES) 2003-2012. Br J Nutr 114, 1294-1303.

58. Murakami K, Miyake Y, Sasaki S et al. (2012) Characteristics of under- and over-reporters of energy intake among Japanese children and adolescents: the Ryukyus Child Health Study. Nutrition 28, 532-538.

59. Troiano RP, Berrigan D, Dodd KW et al. (2008) Physical activity in the United States measured by accelerometer. Med Sci Sports Exerc 40, 181-188.

60. Belcher BR, Berrigan D, Dodd KW et al. (2010) Physical activity in US youth: effect of race/ethnicity, age, gender, and weight status. Med Sci Sports Exerc 42, 2211-2221. 